\title{
Novel conopeptides of the I-superfamily occur in several clades of cone snails
}

\author{
Silke Kauferstein ${ }^{\mathrm{a}, *}$, Isabelle Huys ${ }^{\mathrm{b}}$, Ulrich Kuch ${ }^{\mathrm{a}}$, Christian Melaun ${ }^{\mathrm{a}}$, \\ Jan Tytgat ${ }^{\mathrm{b}}$, Dietrich Mebs ${ }^{\mathrm{a}}$ \\ ${ }^{\mathrm{a}} Z$ Zentrum der Rechtsmedizin, University of Frankfurt, Kennedyallee 104, D-60596 Frankfurt am Main, Germany \\ ${ }^{\mathrm{b}}$ Laboratory of Toxicology, University of Leuven, B-3000 Leuven, Belgium
}

Received 2 February 2004; accepted 6 July 2004

\begin{abstract}
The I-superfamily of conotoxins represents a new class of peptides in the venom of some Conus species. These toxins are characterized by four disulfide bridges and inhibit or modify ion channels of nerve cells. When testing venoms from 11 Conus species for a functional characterization, blocking activity on potassium channels (like Kv1.1 and Kv1.3 channels, but not Kv1.2 channels) was detected in the venom of Conus capitaneus, Conus miles, Conus vexillum and Conus virgo. Analysis at the cDNA level of these venoms using primers designed according to the amino acid sequence of a potassium channel blocking toxin (ViTx) from C. virgo confirmed the presence of structurally homologous peptides in these venoms. Moreover, peptides belonging to the I-superfamily, but with divergent amino acid sequences, were found in Conus striatus and Conus imperialis. In all cases, the sequences of the precursors' prepro-regions exhibited high conservation, whereas the sequences of the mature peptides ranged from almost identical to highly divergent between species. We then performed phylogenetic analyses of new and published mitochondrial 16S rDNA sequences representing 104 haplotypes from these and numerous other Conus species, using Bayesian, maximum-likelihood, maximum-parsimony and neighbor-joining methods of inference. Cone snails known to possess I-superfamily toxins were assigned to five different major clades in all of the resulting gene trees. Moreover, I-superfamily conopeptides were detected both in vermivorous and piscivorous species of Conus, thus demonstrating the widespread presence of such toxins in this speciose genus beyond evolutionary and ecological groups.
\end{abstract}

(C) 2004 Elsevier Ltd. All rights reserved.

Keywords: Conotoxin; I-superfamily; Potassium channel; Mitochondrial DNA; 16S rDNA; Phylogenetic analysis

\section{Introduction}

Venoms from the predatory cone snails (genus Conus) contain numerous disulfide-rich peptides (conotoxins) which exhibit a wide variety of biological activities. Voltage- or ligand-gated ion channels are the main targets of these peptides (Olivera et al., 1991; Olivera and Cruz, 2001).

\footnotetext{
* Corresponding author. Tel.: +49-69-6301-7564; fax: +49-696301-5882.

E-mail address: kauferstein@em.uni-frankfurt.de (S. Kauferstein).
}

Disulfide bridges provide the structural scaffold of conotoxins. The number of cysteine residues, the arrangement of the disulfide bonds and a typical consensus signal sequence are characteristic for the various conotoxin families, which are groups of peptides so far identified in the venoms. The high structural conservation of the disulfides in each conotoxin family is in contrast with the hypervariability of the amino acid sequences in the intercysteine loops (McIntosh et al., 1999; Olivera et al., 1999). The latter results in a high diversity of conotoxins that are able to target various receptors and ion channels (Walker et al., 1999). The discovery of new conotoxins provides valuable tools to 
identify distinct subtypes of ion channels. For instance, the recently described new I-superfamily of conotoxins (Jimenez et al., 2003; Kauferstein et al., 2003) appears to be a promising source for molecular probes to study the most diverse group of ion channels, the potassium channels.

From the venom of the cone snail Conus virgo, a peptide named ViTx (virgo-toxin) was isolated which blocks $\mathrm{K}^{+}$. channels of the Kv1.1 and Kv1.3, but not of Kv1.2 type (Kauferstein et al., 2003). This conotoxin is composed of a chain of 35 amino acid residues cross-linked by four disulfide bridges. Another peptide isolated from Conus betulinus modulates the activity of the BK-potassium channel (Fan et al., 2003), and the venom of Conus radiatus contains peptides of the same family which act on peripheral axons (Jimenez et al., 2003). These findings suggest that the new I-superfamily of conopeptides may be equal in molecular diversity, for instance, to the $\omega$-conotoxins and $\alpha$-conotoxins, which inhibit calcium channels and block acetylcholine receptors, respectively (Terlau and Olivera, 2004).

The present study was initiated to identify similar peptides in the venom of other Conus species by screening crude venoms using electrophysiological testing and PCR analysis of cDNA from their venom glands. In addition, we performed phylogenetic analyses of new and published $16 \mathrm{~S}$ ribosomal DNA (rDNA) sequences using Bayesian, maximum-likelihood (ML), maximum-parsimony (MP) and neighbor-joining (NJ) methods of phylogenetic inference, to elucidate the relationships of cone snails possessing such peptides. Our data reveal that, despite the hypervariability of Conus toxins, members of this particular peptide family may occur with almost identical structural entities and biological activities in the venoms of distantly related Conus species, and that toxins of the I-superfamily are not clade-specific, but distributed widely among several major clades of cone snails, including both vermivorous and piscivorous species.

\section{Materials and methods}

\subsection{Cone snail venoms and tissues}

Venom samples and tissues from specimens of 11 Conus species (C. capitaneus, C. circumcisus, C. imperialis, C. miles, C. mustelinus, C. omaria, C. planorbis, C. striatus, C. textile, C. vexillum, $C$. virgo) were collected around Olango and Bohol Islands, Philippines. The venom apparatus of the snails was dissected and the venom duct was cut into small pieces. The crude venom was extracted with $5 \%$ acetic acid. After centrifugation of the extract at $13,000 \mathrm{rpm}$ for $10 \mathrm{~min}$, the supernatant was lyophilized and stored at $-20{ }^{\circ} \mathrm{C}$ until use. For sequence analysis of $16 \mathrm{~S}$ rDNA and toxin-coding cDNAs, a piece of the proboscis and from the venom duct, respectively, was placed in RNAlater ${ }^{\mathrm{TM}}$ (Ambion Inc., Austin, USA), a tissue storage reagent which protects and stabilizes RNA. Additional species of Conus from the same localities that were used for $16 \mathrm{~S}$ rDNA sequence analysis only are listed below (2.4). Selected vouchers of the cone snail species used in this study were identified conchologically by D. Röckel (pers. comm.). These were used as reference specimens. Taxonomy follows Röckel et al. (1995) except where names differing from the taxonomic concepts in Röckel et al. (1995) had been used in sequence database entries. In such cases, the latter were adopted for the sequences concerned. Information on diet types and main diet taxa of cone snails was obtained from Röckel et al. (1995) and Duda et al. (2001).

\subsection{Venom fractionation}

Lyophilized venom samples (50-100 mg venom gland extract) were dissolved in $0.1 \mathrm{M}$ ammonium acetate, $\mathrm{pH} 6.8$. After centrifugation, the supernatant was subjected to gel filtration on a Sephadex G-50 column $(85 \times 1.5 \mathrm{~cm}$; Amersham Biotech, Budinghamshire, UK) which was equilibrated and eluted with $0.1 \mathrm{M}$ ammonium acetate, $\mathrm{pH}$ 6.8. Fractions of $5 \mathrm{ml}$ were collected at a flow rate of $0.5 \mathrm{ml} / \mathrm{min}$ and lyophilized. After electrophysiological screening, active fractions were dissolved in $0.1 \%$ trifluoroacetic acid and further purified by reversed-phase HPLC on a BDS-Hypersil ${ }^{\circledR}-\mathrm{C}_{18}$-column $(125 \times 4 \mathrm{~mm}$; Hewlett Packard, Waldbronn, Germany). The peptides were eluted with a gradient of trifluoroacetic acid $(0.1 \%$ in water, solvent A) and acetonitrile (60\% in $0.1 \%$ trifluoroacetic acid, solvent B) at a flow rate of $0.5 \mathrm{ml} / \mathrm{min}$ over $45 \mathrm{~min}$. Absorbance was monitored at $220 \mathrm{~nm}$ and the peaks collected were lyophilized and tested for biological activity. Active fractions were rechromatographed using the same conditions, but over a period of $90 \mathrm{~min}$.

\subsection{Electrophysiology}

Analysis of the activity of crude venom and venom fractions towards $\mathrm{K}^{+}$-channels was performed using the Xenopus expression system as described previously (Kauferstein et al., 2003).

Oocytes from Xenopus laevis were prepared as described (Stühmer, 1992). Stage V-VI X. laevis oocytes were isolated by partial ovariectomy. Animals were anaesthetized with tricaine (1 g/l, Sigma, Belgium) and kept on ice during dissection. The vitelline membrane of the oocytes was removed by treatment with $2 \mathrm{mg} / \mathrm{ml}$ collagenase (Sigma, Belgium) in zero calcium ND-96 solution (see below). Capped cRNAs encoding various ion channels (Kv1.1. $\mathrm{Kv1.2,Kv1.3)} \mathrm{to} \mathrm{be} \mathrm{tested} \mathrm{were} \mathrm{synthesized} \mathrm{by} \mathrm{a} \mathrm{standard}$ protocol (Krieg and Melton, 1987). Following the injection of $50 \mathrm{nl}$ of $1-100 \mathrm{ng} / \mu \mathrm{l} \mathrm{cRNA}$, the oocytes were incubated in ND-96 solution at $18{ }^{\circ} \mathrm{C}$ for $1-4$ days to allow the expression of the protein. Lyophilized venom fractions were dissolved in the ND-96 solution containing (in $\mathrm{mM}$ ) 96 $\mathrm{NaCl}, 2 \mathrm{KCl}, 1.8 \mathrm{CaCl}_{2}, 1 \mathrm{MgCl}_{2}, 5$ Hepes, $\mathrm{pH} 7.5$, supplemented with $50 \mathrm{mg} \mathrm{l}^{-1}$ gentamycine sulfate (only for incubation), freshly diluted to the final concentration and added to the bath chamber (volume $100 \mu \mathrm{l}$, perfusion 
rate $5 \mathrm{ml} / \mathrm{min}$ ). All experiments were performed at room temperature $\left(19-22^{\circ} \mathrm{C}\right)$. Currents were obtained using the two-microelectrode voltage clamp technique (Axon Instruments, USA). $\mathrm{K}^{+}$currents were elicited by depolarizations to $0 \mathrm{mV}$ for $100 \mathrm{~ms}$ from a holding potential of $90 \mathrm{mV}$. Current recordings were sampled at $2 \mathrm{kHz}$ after low pass filtering at $0.1 \mathrm{kHz}$. A pulse frequency of $0.2 \mathrm{~Hz}$ was chosen to maximize the recovery from inactivation of the channels. Wash-in experiments were performed by application of $10 \mu \mathrm{g}$ crude venom each to the bath solution.

\subsection{Sequence analysis of $16 \mathrm{~S}$ rDNA}

Tissue from the proboscis of the cone snails was placed in $600 \mu \mathrm{l}$ of lysis buffer $(10 \mathrm{mM}$ Tris, $10 \mathrm{mM}$ EDTA, $100 \mathrm{mM}$ $\mathrm{NaCl}, 2 \% \mathrm{SDS}, \mathrm{pH} \mathrm{7.8-8.0)}$ and digested with $0.4 \mathrm{mg}$ of proteinase $\mathrm{K}$ at $56{ }^{\circ} \mathrm{C}$ overnight, followed by a standard phenol/chloroform extraction (Sambrook and Russell, 2001). Quality and yield of DNA was analyzed by ethidium bromide staining and agarose gel-electrophoresis. PCR amplification of a 16S rRNA gene segment was performed using primers and PCR conditions according to Espiritu et al. (2001). The PCR reaction mixture contained 10 pmol of each primer, $0.2 \mathrm{nM}$ of each dNTP, PCR buffer, $1 \mathrm{U}$ Taq polymerase, and various concentrations of DNA in a total volume of $20 \mu \mathrm{l}$.

Cycle sequencing was performed using Perkin-Elmer Big-Dye Terminator v3.0 reaction premix (Applied Biosystems, Foster City, CA, USA) and the amplification primers. Cycle parameters were the following: 27 cycles of $96^{\circ} \mathrm{C}, 1 \mathrm{~s}$; $50{ }^{\circ} \mathrm{C}, 5 \mathrm{~s} ; 55^{\circ} \mathrm{C}, 4 \mathrm{~min}$; with final ramping to $4{ }^{\circ} \mathrm{C}$. After ddNTP and primer removal using Qiagen dye terminator removal spin columns according to the manufacturer's protocol, the products of the sequencing reaction were analyzed using ABI Model 310 and 3100 automated sequencers (Applied Biosystems, Foster City, CA, USA). The sequences obtained were assembled and analyzed using DNA Sequencing Software 2.1.2 and Sequence Navigator 1.0.1 (Perkin-Elmer Applied Biosystems). The sequences were aligned by eye and against published $16 \mathrm{~S}$ rDNA sequences of Conus spp. and Terebra spp. The alignment is available on request. The following new $16 \mathrm{~S}$ rDNA sequences of Conus spp. were generated in the course of this study (numbers following species names correspond to taxon label designations in Figs. 5 and 6; in parentheses: DDBJ/EMBL/GenBank Nucleotide Sequence Database accession numbers: ammiralis 1 (AJ717586), bandanus 1 (AJ717587), capitaneus 1 (AJ717588), carinatus (AJ717589), circumcisus 1 (AJ746181), flavidus 1 (AJ746182), generalis 1 (AJ717590), geographus 1 (AJ717591), imperialis 1 (AJ717592), imperialis 2 (AJ717593), litteratus (AJ717594), litoglyphus 1 (AJ717595), miles 1 (AJ717596), miles 2 (AJ717597), muriculatus 1 (AJ717599), mustelinus 1 (AJ717600), omaria 1 (AJ717601), omaria 2 (AJ717602), planorbis 1 (AJ746183), quercinus 1 (AJ717603), quercinus 2 (AJ717604), sp. (AJ717598), striatellus 1 (AJ717605), striatellus 3 (AJ717606), striatus 1 (AJ717607), tessulatus 1 (AJ717608), tessulatus 2 (AJ717609), textile 1 (AJ717610), vexillum 1 (AJ717611), vexillum 2 (AJ717612), virgo 1 (AJ717613), vulpinus (AJ717614).

The sequences of Terebra crenulata (AF108825), T. subulata (AF108826), and the following taxa of Conus were obtained from GenBank (numbers following species names are ours, see above): acutangulus (AF160718), ammiralis 2 (AF144000), araneosus (AF174142), arenatus 1 (AF103817), arenatus 2 (AF160700), aulicus (AF126015), aureus (AF108824), bandanus 2 (AF036531), betulinus (AF143999), bullatus (AF126016), californicus (AF036534), capitaneus 2 (AF160701), capitaneus 3 (AF126014), caracteristicus (AF126017), circumactus (AF144001), circumcisus 2 (AF144002), coffeae (AF160703), consors (AF160721), corallinus (AF143995), coronatus (AF126019), ebraeus (AF086613), emaciatus (AF126018), episcopatus (AF126166), ermineus (AF036530), figulinus (AF160702), flavidus 2 (AF160704), frigidus (AF160706), generalis 2 (AF160722), geographus 2 (AF126165), glans (AF126167), gloriamaris (AF126168), granum (AF126169), imperialis 3 (AF108828), litoglyphus 2 (AF144003), lividus (AF086611), loroisii 1 (AF126171), magus 1 (AF086612), magus 2 (AF160707), marmoreus (AF086615), memiae (AF160723), miles 3 (AF108821), miliaris (AF143998), monachus (AF126172), muriculatus 2 (AF160708), musicus (AF144004), mustelinus 2 (AF160709), nigropunctatus (AF086614), nussatella (AF160710), obscurus (AF126173), omaria 3 (AF108823), parvulus (AF143991), pertusus (AF108827), planorbis 2 (AF143996), pulicarius (AF143992), radiatus (AF036529), rattus (AF036533), regius (AF160725), shikamai (AF160720), sponsalis (AF143993), stercusmuscarum (AF103813), striatellus 2 (AF143994), striatus 2 (AF103814), sulcatus (AF160714), terebra (AF103815), tessulatus 3 (AF160715), textile 2 (AF103816), tribblei (AF160716), varius (AF126174), vexillum 3 (AF160717), vexillum 4 (108822), viola (AF160719), virgo 2 (AF086616).

Sequence identity and taxonomic congruence was assessed systematically using BLASTN 2.2.8 (Altschul et al., 1997) in comparing our new sequences to previous submissions. Where two or more $16 \mathrm{~S}$ rDNA sequences had been entered into the database for a single species by different research groups, these were also compared to each other. With only a single exception, all of our $16 \mathrm{~S}$ rDNA sequences of Conus spp. exhibited highest similarities to sequences previously submitted under the same species name. The exception is the sequence of an unidentified Conus species which is conchologically similar to C. magus, but genetically divergent from any cone snail whose sequence was available for comparison. It is here referred to as Conus sp.

In cases of obvious conflict between taxon designations of database submissions by other groups (i.e. incongruence beyond the level of forms or subspecies in apparent species complexes), the taxon involved was not used for analysis. 


\subsection{Phylogenetic inference}

The best-fitting model of molecular evolution was assigned to the mitochondrial data using Modeltest 3.06 (Posada and Crandall, 2001) implemented with PAUP*4.0 (Swofford, 2001). Bayesian Markov Chain Monte Carlo phylogenetic inference (Yang and Rannala, 1997) was performed with MrBayes v.2.01 (Huelsenbeck and Ronquist, 2001) using the best-fit model indicated by Modeltest. Substitution model parameters were estimated as part of the analysis. Three heated chains and one cold chain were initiated with a random starting tree and run for 500,000 generations, saving the current tree every 10 generations. Samples prior to conversion to stationarity of the likelihood sum scores were discarded. The topologies of the last 5000 trees were used to construct a 50\%-majority rule consensus tree using PAUP*4.0, with the percentage of samples that recovered each clade representing posterior clade probabilities.

ML, MP and NJ analyses were performed using PAUP*4.0. The best-fitting substitution model and parameters determined by Modeltest were assumed for ML and $\mathrm{NJ}$ analyses. MP analyses were performed as heuristic searches with 10 random sequence-addition replicates. Support values for clades in NJ analyses were calculated from 2000 bootstrap pseudo-replicates. Gaps were treated as missing data in all analyses.

\section{6. cDNA cloning of conopeptide precursors from the I-superfamily}

To identify cone snail venom gland cDNAs encoding I-superfamily conopeptide precursors, $3^{\prime}$ and $5^{\prime}$ rapid amplification of cloned ends (RACE) experiments were carried out. Two micrograms of RNA were converted into cDNA using superscript 2 reverse transcriptase. The sequence of the conotoxin ViTx (Kauferstein et al., 2003) was used to design primers for use in $3^{\prime}$ and $5^{\prime}$ RACE amplification of toxin-coding cDNAs in other Conus species. $3^{\prime}$ RACE was performed according to Frohman et al. (1989). The $3^{\prime}$ RACE forward primer (5'-ATGATGTTTCGATTGACGTCAGTCAGC- $3^{\prime}$ ) was based on the first part of the peptide sequence MMFRLTSVS of ViTx. PCR conditions were as follows: $95{ }^{\circ} \mathrm{C}, 10 \mathrm{~min}(1 \mathrm{cycle}) ; 95^{\circ} \mathrm{C}, 1 \mathrm{~min} ; 48{ }^{\circ} \mathrm{C}, 1 \mathrm{~min} ; 72{ }^{\circ} \mathrm{C}$, 1 min (30 cycles); $72{ }^{\circ} \mathrm{C}, 7 \mathrm{~min}$ ( 1 cycle). $5^{\prime}$ RACE was performed using first strand cDNA transcripted with the SMART system (Clontech, Palo Alto, USA). The $5^{\prime}$ RACE reverse primer ( $5^{\prime}$-GGAATGTCGCCCTCTTTCCAA- $\left.3^{\prime}\right)$ was based on the peptide sequence GKRATFQ. The PCR protocol consisted of an initial denaturation at $95^{\circ} \mathrm{C}$ for $10 \mathrm{~min}$ followed by $30-40$ cycles at $95{ }^{\circ} \mathrm{C}, 1 \mathrm{~min} ; 48^{\circ} \mathrm{C}, 1 \mathrm{~min} ; 72{ }^{\circ} \mathrm{C}$, $1 \mathrm{~min}$; then 1 cycle of $72{ }^{\circ} \mathrm{C}, 7 \mathrm{~min}$. PCR products of the appropriate size were ligated into the T-tailed plasmid vector pGEM $^{\circledR}$-T (Promega, Madison, USA) and transformed into competent Escherichia coli. Positive clones containing inserts of the expected size were selected for cycle sequencing as described above. The novel cDNA sequences have been deposited in the DDBJ/EMBL/GenBank Nucleotide Sequence Database under the following accession numbers: $C$. imperialis clone 1 (AJ746186), C. imperialis clone 2 (AJ746185), $C$. imperialis clone 3 (AJ746184), C. capitaneus (AJ746187), $C$. striatus (AJ746188), C. miles (AJ746189), C. vexillum clone 1 (AJ748254) and $C$. vexillum clone 2 (AJ748255). The accession number of ViTx is AJ560778.

\section{Results}

\subsection{Screening of Conus venoms for channel blocking activity}

Venoms from eleven Conus species were tested for blocking activity towards several ion channels such as Kv1.1, Kv1.2, Kv1.3, HERG, Kir2.1 and Nav1.5 expressed in Xenopus oocytes using the voltage clamp technique. Three species, C. capitaneus, $C$. miles and $C$. vexillum, were shown to contain ion channel inhibiting activities in their venoms (Fig. 1). Further purification of the peptide(s) involved was attempted using gel filtration of the crude
Conus miles

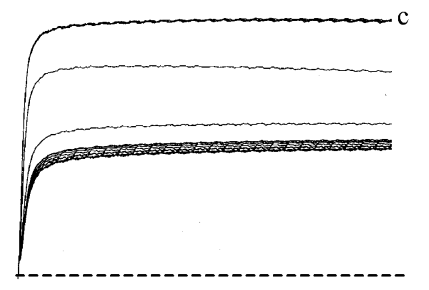

$1 \mu \mathrm{A}$
Conus vexillum

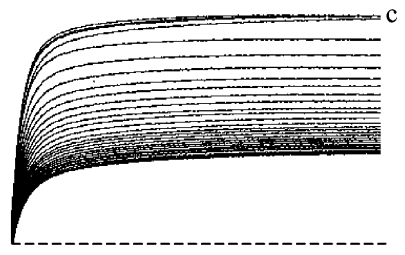

Conus capitaneus

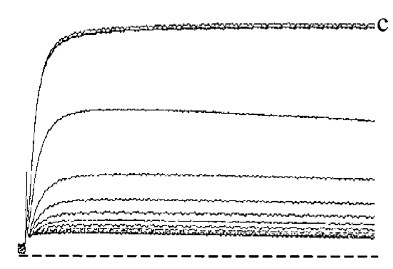
$10 \mathrm{~ms}$

Fig. 1. Macroscopic $\mathrm{K}^{+}$current through potassium Kv1.1 channel expressed in Xenopus oocytes. Oocytes were clamped at a holding potential of $-90 \mathrm{mV}$ and stepped to $0 \mathrm{mV}$ for $100 \mathrm{~ms}$. After application of the venoms of C. miles, C. vexillum and C. capitaneus, respectively, currents through Kv1.1 channels were recorded. $\mathrm{c}=$ control. 

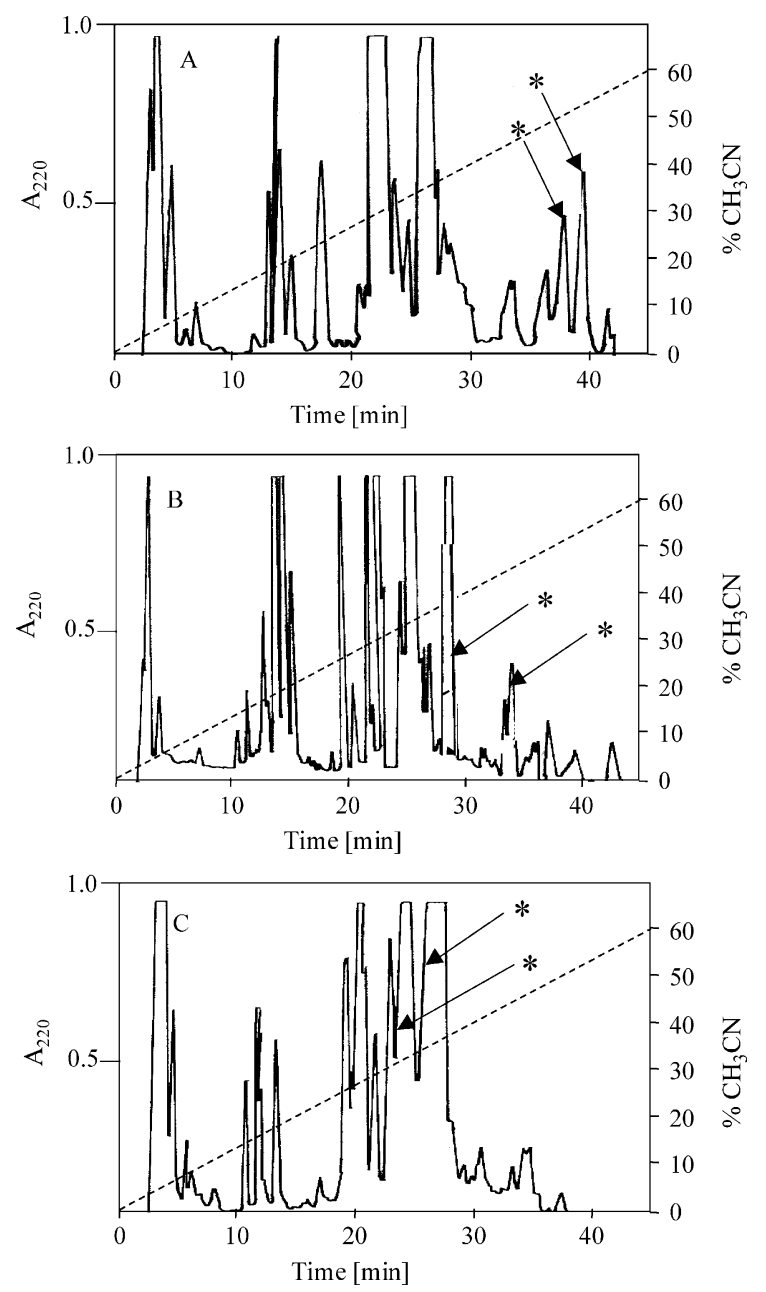

Fig. 2. Fractionation of venoms from cone snails by HPLC. The active second fractions from the gel filtration (Sephadex G-50) of the venoms from C. miles (A), C. vexillum (B) and C. capitaneus (C) were further separated by reversed-phase HPLC on a BDSHypersil- $\mathrm{C}_{18}$-column $(125 \times 4 \mathrm{~mm})$ and eluted with a linear gradient from 0 to $60 \%$ acetonitrile in $0.1 \%$ trifluoroacetic acid over $45 \mathrm{~min}$ at a flow rate of $0.5 \mathrm{ml} / \mathrm{min}$. Absorbance was monitored at $220 \mathrm{~nm}$. The active fractions are indicated by arrows.

venoms on Sephadex G-50 (2nd fraction) followed by reversed phase HPLC (Fig. 2). The active fractions had essentially the same properties as the peptide ViTx from the venom of $C$. virgo, i.e. blocking $\mathrm{Kv} 1.1$ and $\mathrm{Kv} 1.3 \mathrm{~K}^{+}$channels, but not Kv 1.2 channels (Kauferstein et al., 2003). However, a further structural characterization of the peptides in these venoms was not possible due to the very low yield of the active peptide(s) present in these venoms.

\section{2. cDNA cloning of conopeptide precursors from the I-superfamily}

Multiple clones were isolated and sequenced. At least one precursor sequence was obtained for each Conus species. The sequences of the open reading frame were aligned to maximize sequence similarity. Sequence analysis of the clones revealed that they all share the common features of conotoxins such as a highly conserved sequence of the signal peptide and a variable mature toxin region. With eight cysteine residues arranged in a well-defined pattern (Fig. 3), these peptides belong to the previously described I-conotoxin superfamily like ViTx from C. virgo (Kauferstein et al., 2003; Jimenez et al., 2003). After inferring boundaries of sequences encoding signal peptides and mature toxins from the known sequence of ViTx, resulting in two alignments of 78 and $108 \mathrm{bp}$, respectively, sequence comparison revealed very high similarity of the signal peptide region (Fig. 4A). An identical sequence was shared between the cDNA of ViTx and the two obtained from C. vexillum. Apart from a 6 bp deletion that was shared by all $C$. imperialis clones, one of the $C$. imperialis cDNAs (C. imperialis 3) also exhibited the same sequence. Sequence divergence between this and the corresponding segments of the other cDNAs ranged from 1.3-3.8\%, with the exception of $C$. imperialis 1 , which differed from all other clones in 5.1-7.7\%. High sequence variability, on the other hand, was expected for the nucleotide sequences encoding the intercysteine loops of the mature toxins. This expectation was fully met by the cDNAs from $C$. imperialis, which differed from each other and from the other species in $33-52 \%$ of nucleotides in this region (Fig. 4B), and have a 3 bp deletion each. The sequences of $C$. capitaneus, $C$. miles and $C$. striatus differed from those of $C$. virgo and $C$. vexillum in $25.7-27.6 \%$, and from each other in $9.3-16.7 \%$ of sites. Interestingly, the nucleotide sequences of the mature peptide regions of $C$. vexillum were very similar to that of ViTx from C. virgo, differing only in 4.8-5.7\% (5-6 nucleotides) which result in one (C. vexillum 1$)$ and two $(C$. vexillum 2) amino acid substitutions. The two sequences from C. vexillum differ in only $1 \%$ (one nucleotide) in this region, resulting in a single amino acid substitution.

\subsection{Phylogenetic inference}

Alignment of 104 sequences of Conus spp. and one sequence each of $T$. crenulata and $T$. subulata resulted in 513 sites of $16 \mathrm{~S}$ rDNA. Two unalignable hypervariable regions of 15 and 27 nucleotides (positions 181-195 and $257-283$, respectively) were excluded prior to analyses. In the reduced data matrix of 471 sites, 292 were constant. Of 179 variable characters, 139 were informative under the maximum-parsimony optimality criterion. The MP searches resulted in 282 equally parsimonious trees of 1028 steps. The strict consensus of the 282 trees was used for comparison with other phylogenetic hypotheses. Along with the best tree of the ML analysis (-log likelihood 5429.2512), it is not illustrated here (available on request). Fig. 5 shows the 50\%-majority rule consensus tree constructed from the last 5000 trees obtained by Bayesian 

C. virgo:
C. vexilhum (1):
C. vexillum (2):
C. miles :
C. striatus:
C. capitaneus:
C. imperialis (1):
C. imperialis (2):
C. imperialis (3):

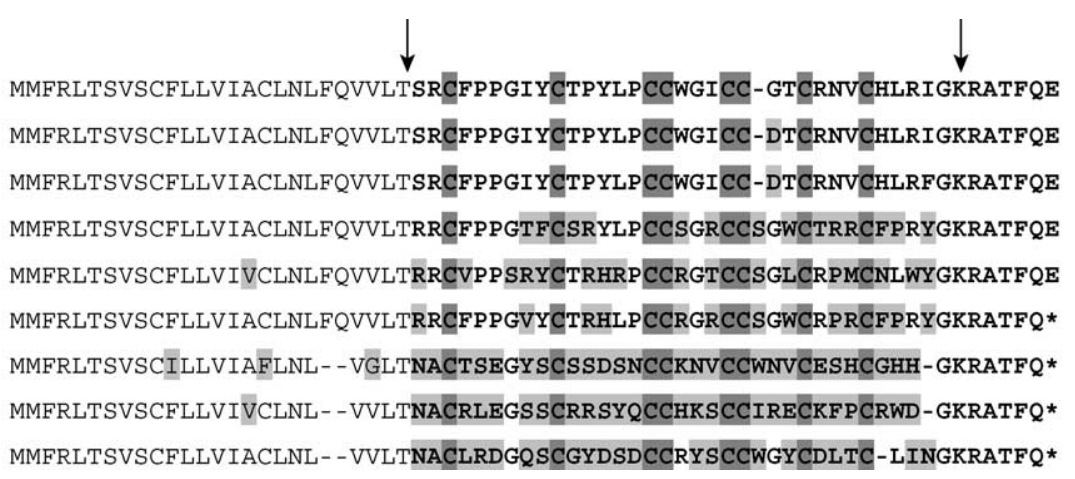

Fig. 3. Deduced amino acid sequences of I-superfamily peptides including their prepro-regions. Conserved cystein patterns are indicated in dark grey, residues differing from the sequence of the $C$. virgo peptide in light grey. Numbers in parentheses designate different peptides from one Conus species. The mature peptide appears in bold print. Posttranslational cleavage sites are indicated by arrows.

inference analysis. Fig. 6 shows the NJ tree obtained for the same data.

Details of the phylogenetic hypotheses obtained using the four methods of inference are discussed below (4.2). Regardless of differences between them, the species of Conus from which toxins of the I-superfamily have been recorded are consistently assigned to five or six major clades of cone snails: (1) C. betulinus is a member of a strongly supported clade of vermivorous species that is variably associated with $C$. obscurus (Bayesian, ML, MP trees; see discussion) or $C$. sp. (NJ); (2) C. capitaneus, $C$. miles and C. vexillum are members of a strongly supported clade of cone snails which prey on eunicid, nereid and spinoid polychaetes and also contains $C$. mustelinus, $C$. pertusus and C. rattus; (3) $C$. striatus is a member of a clade of piscivorous and occasionally molluscivorous cone snails that is present in all four analyses, although with variable clade support and within-clade topologies; (4) the clade containing $C$. radiatus (and $C$. monachus, C. ermineus) is the sister clade of the piscivorous clade (3) in the Bayesian and NJ, but appears in two different positions in the ML and MP trees; (5) C. virgo is a member of a strongly supported clade (with $C$. emaciatus, C. flavidus, C. frigidus, C. terebra) containing cone snails preferring capitellid and terebellid polychaetes as food; (5) Conus imperialis, a predator of amphinomid polychaetes, is identified as one of

\begin{tabular}{|c|c|c|c|c|c|c|c|c|c|}
\hline & C. virgo & C. vexillum (1) & C. vexillum (2) & C. capitaneus & C. miles & C. striatus & C. imperialis (3) & $C$ imperialis $(2)$ & $C$ imperialis (1) \\
\hline C. virgo & - & 0 & 0 & 1.3 & 2.6 & 1.3 & 0 & 13 & 51 \\
\hline C. vexillum (1) & & - & 0 & 1.3 & 2.6 & 1.3 & 0 & 1.3 & 5.1 \\
\hline C. vexillum (2) & & & - & 1.3 & 2.6 & 1.3 & 0 & 1.3 & 5.1 \\
\hline C. capitaneus & & & & - & 3.8 & 2.6 & 1.3 & 2.6 & 6.4 \\
\hline C. miles & & & & & - & 3.8 & 2.6 & 3.8 & 7.7 \\
\hline C. striatus & & & & & & - & 1.3 & 0 & 6.4 \\
\hline C. imperialis (3) & & & & & & & - & 1.3 & 5.1 \\
\hline C. imperialis (2) & & & & & & & & - & 6.4 \\
\hline C. imperialis (1) & & & & & & & & & - \\
\hline
\end{tabular}

B

\begin{tabular}{|c|c|c|c|c|c|c|c|c|c|}
\hline & C. virgo & C. vexillum (1) & C. vexillum (2) & C. capitaneus & C. miles & C. striatus & C. imperialis (3) & C. imperialis (2) & C. imperialis (1) \\
\hline C. virgo & - & 4.8 & 5.7 & 25.7 & 26.7 & 26.7 & 46.1 & 40.2 & 49.0 \\
\hline C. vexillum (1) & & - & 1.0 & 26.7 & 27.6 & 27.6 & 47.1 & 40.2 & 52.0 \\
\hline C. vexillum (2) & & & - & 25.7 & 26.7 & 26.7 & 48.0 & 40.2 & 52.0 \\
\hline C. capitaneus & & & & - & 9.3 & 13.0 & 42.9 & 39.0 & 51.4 \\
\hline C. miles & & & & & - & 16.7 & 42.9 & 39.0 & 48.6 \\
\hline C. striatus & & & & & & - & 45.7 & 41.0 & 52.4 \\
\hline C. imperialis (3) & & & & & & & - & 33.3 & 39.0 \\
\hline C. imperialis (2) & & & & & & & & - & 40.0 \\
\hline C. imperialis (1) & & & & & & & & & - \\
\hline
\end{tabular}

Fig. 4. Percent divergence of the nucleotide sequences of the prepro-region (A) and of the mature toxin region (B; see 3.2 for definitions) of the novel I-superfamily conopeptides and ViTx from $C$. virgo. 


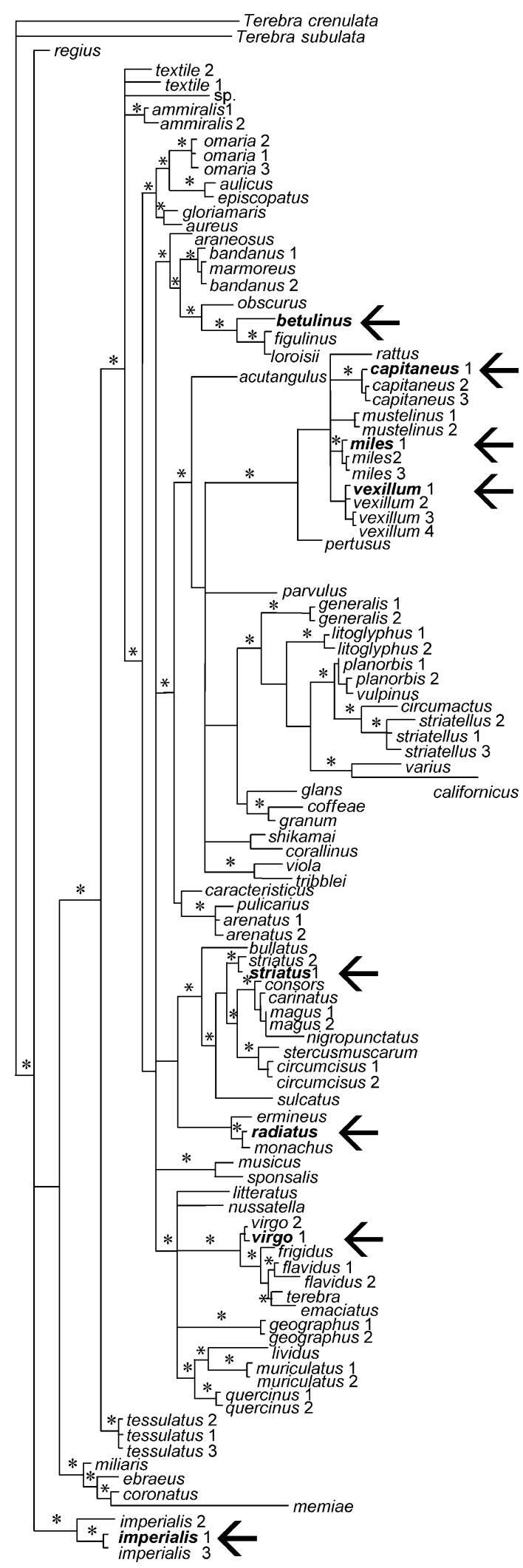

the few most basal taxa by the Bayesian, NJ and MP analyses (strongly supported in the former two), but has a divergent position in the ML tree.

The presence of toxins belonging to the I-superfamily of conopeptides is thus demonstrated across several major clades in our mitochondrial gene trees. At the same time, I-superfamily conopeptides are shown to be present in cone snails which, with respect to their feeding ecology (Röckel et al., 1995; Duda et al., 2001), may be assigned to three major groups preying on (a) fishes, (b) errant polychaete annelids, or (c) sedentary polychaete annelids.

\section{Discussion}

\subsection{Identification of toxins of the I-superfamily in Conus venoms}

Venoms from cone snails represent a rich source of biologically active peptides. However, the large number of different peptides in a venom may reduce the availability of a particular component due to its low concentration in the venom, or due to the poor yield after several steps of purification. Although biological activity may still be detectable, the amount of pure material obtained might not be sufficient to complete structural studies such as by Edman degradation, particularly when the number of snails used for venom extraction is limited. However, the determination of partial amino acid sequences, followed by cDNA cloning and sequencing, usually allows for the deduction of the complete amino acid sequences (cf. Kauferstein et al., 2003; Fan et al., 2003).

Among the various conotoxins isolated so far, those exhibiting four disulfide bonds represent a new superfamily (I-superfamily; Fan et al., 2003; Jimenez et al., 2003; Kauferstein et al., 2003). Toxins like ViTx from the venom of $C$. virgo show marked inhibitory activity towards vertebrate voltage-sensitive potassium channels, others like $\kappa$-Btx from the venom of $C$. betulinus modulate potassium (BK) channels, or peptides from C. radiatus venom elicit excitatory activity in peripheral axons (Jimenez et al., 2003). Except for the identical positions of cysteine residues, these peptides differ markedly in their amino acid sequences of their intercysteine loops.

In the present study, specific targets, namely the potassium and sodium channels, were used to screen venoms from eleven Conus species for inhibitory activity. Three venoms showed blocking effects on the potassium channels Kv1.1 and 1.3, but not on Kv1.2 channels,

Fig. 5. Bayesian analysis of cone snail 16S rDNA sequences. Posterior clade probabilities greater than $80 \%$ are indicated by asterisks along the internodes $(*=80-94 \% ; *=95-100 \%)$. Arrows and bold print mark those individuals or species from which I-superfamily conopeptides are known. 


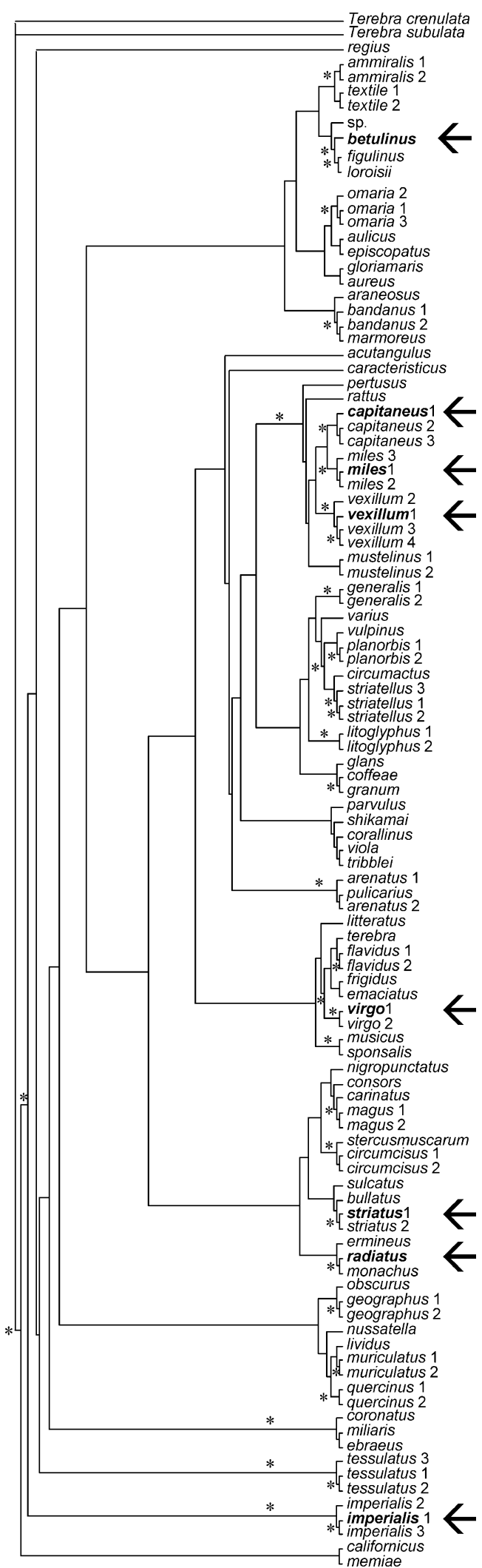

suggesting the presence of peptide(s) which may be similar to ViTx in structure and activity. Other cloned ion channels (HERG channels, Kir2.1 channels and Nav1.5 channels) were also assayed, but no activity could be observed. Although Kv1.1 and 1.3 blocking activity was identified in a particular venom fraction, the material obtained was neither pure enough nor available in sufficient quantities to perform structural studies by Edman degradation or even massspectrometry analysis. However, genetic analysis using primers designed according to the amino acid sequence of ViTx confirmed the presence of similar to almost identical peptides and elucidated the sequences of their preproregions. Since posttranslational cleavage of a six-residue peptide from the C-terminus had been demonstrated in the case of ViTx from the venom of C. virgo (Kauferstein et al., 2003), similar modifications were assumed for the novel peptides. Indeed, two isotoxins which differ from ViTx in only a single amino acid residue were detected in C. vexillum, and others of close structural similarity in C. miles and C. capitaneus. Since the primer used in this experiment also included the prepro-region of the toxin precursor, genetic analysis revealed other toxins belonging to the I-superfamily in $C$. striatus and $C$. imperialis. These exhibited a highly divergent primary structure in the intercysteine sections suggesting still unknown physiological activities. The elucidation of the nucleotide sequences of these latter cDNAs and their deduced amino acid sequences suggests that the known phenomenon of hypervariable toxin sequences coupled with conserved prepro-regions may also occur in the I-superfamily of conopeptides. Nevertheless, the observed percent divergence of the prepro-regions (up to $7.7 \%$ ) was much lower than that reported for the $\delta$-conotoxins (up to $17.8 \%$; Espiritu et al., 2001).

We were thus surprised to also document the presence of almost identical or similar peptides in venoms from Conus species which are morphologically distinct, and only distantly related according to our phylogenetic analysis (Figs. 5 and 6). However, this is not without precedence, since Nicke et al. (2003) reported an $\alpha 4 / 7$ conotoxin (GID) from the venom of Conus geographus whose amino acid sequence is identical to that in the venom of Conus tulipa. Further studies are required to investigate the evolutionary processes underlying the apparent lack of conotoxin variability in these cases. In particular, the question deserves attention whether the observed high sequence similarity reflects introgression among sympatric Conus species, stabilizing molecular evolution of an ancient toxin, or is the result of hypervariable toxins attaining nearly identical sequences by other molecular mechanisms, chance, or convergence.

Fig. 6. Neighbor-joining tree of cone snail 16S rDNA sequences. Asterisks mark clades with $80-100 \%$ bootstrap support. Arrows and bold print mark those individuals or species from which I-superfamily conopeptides are known. 


\subsection{Phylogenetic inference}

In our phylogenetic analyses of mitochondrial $16 \mathrm{~S}$ rDNA sequences from cone snails, many of the terminal clades previously identified by Duda et al. (2001) and Espiritu et al. (2001) were also recovered. In particular, there is congruence between studies and hypotheses based on different methods of phylogenetic inference as to the distinctiveness of (and support for) the clades containing species from which I-superfamily conopeptides are known. The distribution of toxins belonging to the I-superfamily in several major clades is thus strongly supported by the phylogenetic analyses. This includes the basal $C$. imperialis clade (this study, see also below), the $C$. virgo clade (Kauferstein et al., 2003), C. striatus (this study), C. radiatus (Jimenez et al., 2003), the $C$. pertusus clade with three species $(C$. capitaneus, C. miles and $C$. vexillum, this study), and the $C$. betulinus clade (Fan et al., 2003). A notable difference in the topologies of the Bayesian, MP and NJ trees, if compared to previously published gene trees, is the position of the $C$. imperialis clade, which is one of the most basal groups in our analyses and those by Duda et al. (2001). This basal position, which is also supported by morphology (Röckel et al., 1995), was not reflected in ML analyses (Espiritu et al., 2001; this study). Conversely, one of the most basal species in the ML, MP and NJ trees in Duda et al. (2001), Espiritu et al. (2001) and this study, C. californicus, surfaced at a grossly different position in our Bayesian tree. Another intriguing incongruence concerns the relationships of the piscivorous species $C$. obscurus, which is associated with another piscivorous species (C. geographus) in the NJ trees by Duda et al. (2001) and this study, and the ML tree by Espiritu et al. (2001). However, in the Bayesian, ML and MP analyses performed in this study, C. obscurus is nested within a clade containing one clade of vermivorous $(C$. betulinus and relatives) and one of molluscivorous species ( $C$. bandanus, C. marmoreus).

Congruence between relationships of Conus species as predicted by Röckel et al. (1995) based on morphological characters and the topologies of the mitochondrial gene trees is generally high. As expected, this is particularly so in the case of terminal clades. On the other hand, species with unique and greatly divergent morphologies like $C$. imperialis and $C$. ammiralis also have basal positions in the trees.

Differences between the mitochondrial gene trees in this and previous studies may be explained by the different methods of phylogenetic inference employed, and by differences in the alignment of sequences (ours excluding two hypervariable regions of the $16 \mathrm{~S}$ rRNA gene which could not be reliably aligned), choice of outgroup and the number and composition of ingroup taxa. In the future, increased sampling of taxa (Pollock et al., 2002; Zwickl and Hillis, 2002) as well as sequence data per taxon are expected to greatly increase the overall accuracy of phylogenetic estimates for this extraordinarily speciose group of venomous animals.

\section{Conclusion}

Our study demonstrates the widespread presence of I-superfamily toxins in five or six major clades of cone snails, suggesting the possibility that such toxins may eventually be found in many more species. In particular, their occurrence in groups with different feeding ecologies and the basal phylogenetic position of $C$. imperialis lend weight to the notion that toxins of the I-superfamily or their genes may be comparatively ancient and more universally distributed among cone snails than certain other groups of apparently derived, sometimes cladespecific conopeptides.

\section{Acknowledgements}

We thank Olaf Pongs for providing the cDNA for the Kv1.2 channel. The Kv1.3 clone was kindly provided by María L. García. The HERG clone was generously donated by Mark Keating. The Kir2.1 cDNA clone was kindly provided by Lilly Y. Jan. The hH1 clone was kindly provided by Roland G. Kallen. Isabelle Huys is a Research Assistant of the Flemish Fund for Scientific Research (F.W.O.-Vlaanderen). We thank Thomas Heeger for his support during our work in the Philippines, and two anonymous reviewers for their constructive comments. This work was supported in part by a grant of the Deutsche Forschungsgemeinschaft to D.M. and grant G.0081.02 (F.W.O. Vlaanderen) to J.T.

\section{References}

Altschul, S.F., Madden, T.F., Schäffer, A.A., Zhang, J., Zhang, Z., Miller, W., Lipman, D.J., 1997. Gapped BLAST and PSIBLAST: a new generation of protein database search programs. Nucleic Acids Res. 25, 3389-3402.

Duda Jr., T.F., Kohn, A.J., Palumbi, S.R., 2001. Origins of diverse feeding ecologies within Conus, a genus of venomous marine gastropods. Biol. J. Linn. Soc. 73, 391-409.

Espiritu, D.J., Watkins, M., Dia-Monje, V., Cartier, G.E., Cruz, L.J., Olivera, B.M., 2001. Venomous cone snails: molecular phylogeny and the generation of toxin diversity. Toxicon 39, 1899-1916.

Fan, C.X., Chen, X.K., Zhang, C., Wang, L.X., Duan, K.L., He, L.L., Cao, Y., Liu, S.Y., Zhong, M.N., Ulens, C., Tytgat, J., Chen, J.S., Chi, C.W., Zhou, Z., 2003. A novel conotoxin from Conus betulinus, kappa-BtX, unique in cysteine pattern and in function as a specific BK channel modulator. J. Biol. Chem. 278, 12624-12633.

Frohman, M.A., Dush, M.K., Martin, G.R., 1989. Rapid production of full-length cDNAs from rare transcripts: amplification using a single gene-specific oligonucleotide primer. Proc. Natl Acad. Sci. USA 85, 8998-9002.

Huelsenbeck, J.P., Ronquist, F.R., 2001. MrBayes: Bayesian inference of phylogeny. Bioinformatics 17, 754-755. 
Jimenez, E.C., Shetty, R.P., Lirazan, M., Rivier, J., Walker, C., Abogadie, F.C., Yoshikami, D., Cruz, L.J., Olivera, B.M., 2003. Novel excitatory Conus peptides define a new conotoxin superfamily. J. Neurochem. 85, 610-621.

Kauferstein, S., Huys, I., Lamthanh, H., Stöcklin, R., Sotto, P., Ménez, A., Tytgat, J., Mebs, D., 2003. A novel conotoxin inhibiting vertebrate voltage-sensitive potassium channels. Toxicon 42, 43-52.

Krieg, P.A., Melton, D.A., 1987. In vitro RNA synthesis with SP6 RNA polymerase. Methods Enzymol. 155, 397-441.

McIntosh, M.J., Santos, A.D., Olivera, B.M., 1999. Conus peptides targeted to specific nicotinic acetylcholine receptor subtypes. Annu. Rev. Biochem. 68, 59-88.

Nicke, A., Loughnan, M.L., Millard, E.L., Alewood, P.F., Adams, D.J., Daly, N.L., Craik, D.J., Lewis, R.J., 2003. Isolation, structure, and activity of GID, a novel alpha 4/7-conotoxin with an extended N-terminal sequence. J. Biol. Chem. 278, 3137-3144.

Olivera, B.M., Cruz, L.J., 2001. Conotoxins, in retrospect. Toxicon 39, 7-14.

Olivera, B.M., Rivier, J., Scott, J.K., Hillyard, D.R., Cruz, L.J., 1991. Conotoxins. J. Biol. Chem. 266, 22067-22070.

Olivera, B.M., Walker, C., Cartier, G.E., Hooper, D., Santos, A.D., Schoenfeld, R., Shetty, R., Watkins, M., Bandyopadhyay, B., Hillyard, D.R., 1999. Speciation of cone snails and interspecific hyperdivergence of their venom peptides: potential evolutionary significance of introns. Ann. NY Acad. Sci. 870, 223-237.
Pollock, D.D., Zwickl, D.J., McGuire, J.A., Hillis, D.M., 2002. Increased taxon sampling is advantageous for phylogenetic inference. Syst. Biol. 51, 664-671.

Posada, D., Crandall, K.A., 2001. Selecting the best-fit model of nucleotide substitution. Syst. Biol. 50, 580-601.

Röckel, D., Korn, W., Kohn, A.J., 1995. Manual of the Living Conidae, Vol. I: Indo-Pacific Region. Verlag Christa Hemmen, Wiesbaden, Germany.

Sambrook, J., Russell, D.W., 2001. Molecular Cloning: a Laboratory Manual, third ed. Cold Spring Harbor Laboratory Press, Cold Spring Harbor, NY, USA.

Stühmer, W., 1992. Electrophysiological recording from Xenopus oocytes. Methods Enzymol. 207, 319-339.

Swofford, D.L. 2001. PAUP: phylogenetic analysis using parsimony (* and other methods). Version 4.0. Sinauer, Sunderland, MA, USA.

Terlau, H., Olivera, B.M., 2004. Conus venoms: a rich source of novel ion channel-targeted peptides. Physiol. Rev. 84, 41-68.

Walker, C., Steel, D., Jacobsen, R.B., Liranzan, M.B., Cruz, L.J., Hooper, D., Shetty, R., De la Cruz, R.C., Nielsen, J.S., Zhou, L., Bandyopadhyay, P., Craig, A., Olivera, B.M., 1999. The Tsuperfamily of conotoxins. J. Biol. Chem. 274, 30664-30671.

Yang, Z., Rannala, B., 1997. Bayesian phylogenetic inference using DNA sequences: a Markov chain Monte Carlo method. Mol. Biol. Evol. 14, 717-724.

Zwickl, D.J., Hillis, D.M., 2002. Increased taxon sampling greatly reduces phylogenetic error. Syst. Biol. 51, 588-598. 